LAUR-00-3925

SU-4240-722

August 2000

\title{
Phase diagram of four-dimensional dynamical triangulations with a boundary
}

\author{
Simeon Warner*, Simon Catterall ${ }^{\dagger}$ \\ * T-8, Los Alamos National Laboratory, NM 87545, USA \\ $\dagger$ Department of Physics, Syracuse University, Syracuse, NY 13210, USA
}

\begin{abstract}
We report on simulations of DT simplicial gravity for manifolds with the topology of the 4-disk. We find evidence for four phases in a two-dimensional parameter space. In two of these the boundary plays no dynamical role and the geometries are equivalent to those observed earlier for the sphere $S^{4}$. In another phase the boundary is maximal and the quantum geometry degenerates to a one dimensional branched polymer. In contrast we provide evidence that the fourth phase is effectively three-dimensional. We find discontinuous phase transitions at all the phase boundaries.
\end{abstract}

\section{Introduction}

Dynamical triangulation (DT) models arise from simplicial discretizations of continuous Riemannian manifolds. A manifold is approximated by glueing together a set of equilateral simplices with fixed edge lengths. This glueing ensures that each face is shared by exactly two distinct simplices the resultant simplicial lattice is often called a triangulation. In the context of Euclidean quantum gravity it is natural to consider a weighted sum of all possible triangulations as a candidate for a regularized path integral over metrics. Physically distinct metrics correspond to inequivalent simplicial triangulations. This prescription has been shown to be very successful in two-dimensions

\footnotetext{
${ }^{0}$ Corresponding author: Simeon Warner, email: simeon@lanl.gov
} 
in which analytic methods have been complemented by simulation studies. (see, for example, [2]). In dimensions above two there are no known analytic techniques for handling the sum over triangulations and we must rely on numerical simulation. All of the latter studies have focused on elucidating the phase structure of compact manifolds, principally the sphere $S^{4}$. In this paper we investigate the phase structure of four manifolds with the topology of a 4-disk - that is a four sphere $S^{4}$ equipped with a single boundary with topology $S^{3}$. As we will demonstrate the dimension of the parameter space of this model is larger than the corresponding compact models. The simplest compact models exhibit only discontinuous phase transitions precluding a continuum limit. One motivation for the current work was to see whether the richer parameter space of the non-compact models contains any continuous phase transitions. A natural lattice action $S_{b}$ can be derived from the continuum action by straightforward techniques [9]. It contains both the usual Regge curvature piece familiar from compact triangulations together with a boundary term. The boundary term arises from discretization of the extrinsic curvature of the boundary embedded in the bulk. In four-dimensions the curvature is localized on triangles. If $T_{M}$ denotes the set of triangles in the bulk of the 4-triangulation (excluding the boundary) and $T_{\partial M}$ those in the boundary the action can be written

$$
S_{E H}=\kappa_{2}\left(\sum_{h \in T_{M}}\left(2 \pi-\alpha n_{h}\right)+\sum_{h \in T_{\partial M}}\left(\pi-\alpha n_{h}\right)\right)+\kappa_{4} N_{4}
$$

The quantity $\alpha=\arccos (1 / 4), N_{4}$ is the 4 -volume and $n_{h}$ is the number of simplices sharing the triangle (hinge) $h$. The curvature part of the action can be rewritten in terms of the number of vertices $N_{0}$, the boundary volume $N_{3}^{b}$ and the number of boundary vertices $N_{0}^{b}$. With this in mind we shall consider the general simplicial action

$$
S_{b}=-\kappa_{0} N_{0}+\kappa_{4} N_{4}+\kappa_{b} N_{3}^{b}+\kappa_{b}^{0} N_{0}^{b}
$$

This form of the action contains both cosmological constant terms and curvature terms for the bulk and boundary. Notice that the term involving $\kappa_{b}^{0}$ is absent in three dimensions since it is related to the curvature of a boundary two-sphere which is a topological invariant. In this work we have always set $\kappa_{b}^{0}=0$ and $\kappa_{4}$ is used to tune the volume of the 4 -disk. We are thus left with a two-dimensional phase space parameterized by $\kappa_{0}$ and $\kappa_{b}$ conjugate to the number of vertices and 
the number of boundary tetrahedra.

The partition function for the system is then

$$
Z=\sum_{T} e^{-S_{b}}
$$

where the sum is over triangulations, $T$.

\section{Simulation}

Our simulation algorithm is an extension of the algorithm for compact manifolds in arbitrary dimension described by Catterall [5] and is described in [11]. To simulate a triangulation with a boundary we actually simulate a compact triangulation with the topology of the sphere but consider one marked vertex to lie outside the bulk triangulation. This vertex may never be removed during the course of the simulation and the surface of the ball created by its neighbour simplices constitutes the triangulated boundary of the 4-disk. The form of the action (equation 2) can then be derived by writing down an expression for the integrated curvature of the full sphere expressed as contributions from the 4-disk and the simplices around the marked vertex. The latter contributions can be evaluated explicitly in terms of bulk and boundary simplex numbers and yield this simple form for the action. To perform a simulation we need a set of local moves which are ergodic on the space of triangulations of the 4-disk. Fortunately we have such a set of moves - the usual moves on the sphere.

In four-dimensions there are just 5 types of move: vertex insertion, vertex deletion, exchange of a link with a tetrahedron (two moves: link to tetrahedron and tetrahedron to link), and exchange of one triangle for another triangle. Where these moves take place on sections of the triangulation involving the marked vertex we take care to count changes in the numbers of simplices inside and outside the boundary so that we can calculate the change in the action, but otherwise the moves are the same as for the bulk.

The code is described in more detail in [11] where it was used for the simulation of three-dimensional 
dynamical triangulations with a boundary. The code was written for arbitrary dimension and earlier checked against other workers' results in two-dimensions [1].

We have used the Metropolis Monte Carlo [10] scheme with usual update rule:

$$
p(\text { accept move })=\min \left\{e^{-\Delta S_{b}}, 1\right\}
$$

and in this way we explore the space of triangulations with the action $S_{b}$.

\section{Measurements}

Here we define some of the measurements used to characterize the configurations obtained from our simulations. During the simulations we store configurations at some interval which is sufficient to ensure that the configurations are independent. We check this by estimating the auto-correlation time $(\tau)$ of each measurement when calculating expectation values. Uncorrelated data would give $\tau=0.5$, we quote $\tau$ when it significantly exceeds 0.5 .

We use two geodesic measures, $d_{a v g}$ and $d_{b d y}$. The average geodesic distance between simplices, $d_{\text {avg }}$, is measured by counting the smallest number of steps between adjacent simplices required to get from one randomly selected simplex to another randomly selected simplex and taking the mean over a sample of such measurements. The mean geodesic distance to the boundary, $d_{b d y}$, is measured by counting the smallest number of steps between adjacent simplices required to get from one randomly selected simplex to the boundary and taking the mean over a sample of such measurements. The last step from a boundary simplex to the boundary is counted as 0.5.

To discuss the singular vertex structure observed in some phases we will consider a coordination measure, $q$, defined as the number of simplices sharing a given vertex. Similarly we define $\left\langle q_{\max }\right\rangle$ as the expectation value of the coordination of the most highly coordinated vertex; $\left\langle q_{\text {nextMax }}\right\rangle$ as the expectation value of the coordination of the next most highly coordinated vertex; $\left\langle q_{\text {avg }}\right\rangle$ as the expectation value of the mean coordination of all vertices in the triangulation; and $\left\langle q_{b d y}\right\rangle$ as the expectation value of the coordination of the boundary by which we mean the average number of 
unique 4-simplices being shared by a typical boundary vertex.

\section{Phase diagram}

We performed a set of simulations in four dimensions with action of equation 2. In all runs $\kappa_{4}$ was used to tune the nominal system volume, $N_{4}$, for each given $\kappa_{0}$ and $\kappa_{b}$. To map the phase diagram we used $N_{4}=1000$; while to characterize the phases we used $N_{4}=8000$. In order to check the orders of transitions we have also used finite size scaling using additional simulations at $N_{4}=2000$ and $N_{4}=4000$.

Series of runs varying either $\kappa_{0}$ or $\kappa_{b}$ were made and the vertex susceptibility used to search for phase transitions. We define the vertex susceptibility, $\chi$, to be normalized with respect to the number of 4-simplices:

$$
\chi=\frac{1}{N_{4}}\left(\left\langle N_{0}^{2}\right\rangle-\left\langle N_{0}\right\rangle^{2}\right)
$$

The points shown in figure 2 are taken from the positions of peaks in the vertex and boundary susceptibilities.

In figure 2 there are four phases which we characterize as: crumpled, minimal boundary (CMB); branched-polymer, minimal boundary (BPMB); boundary dominated (BD); and intermediate bound$\operatorname{ary}(\mathrm{IB})$.

In $\mathrm{CMB}$ and BPMB phases the boundary is simply 5 tetrahedra (3-simplices) connected to form a hyper-tetrahedral hole. The system is essentially a sphere with one marked 4-simplex — the hyper-tetrahedral hole.

\section{CMB phase}

Here we show typical data for $N_{4}=8000$ at $\kappa_{0}=-4$ and $\kappa_{b}=4$ using 137 samples (a sample corresponds to 100000 attempted updates). The boundary size is just 5 - a minimal hole and we see one 


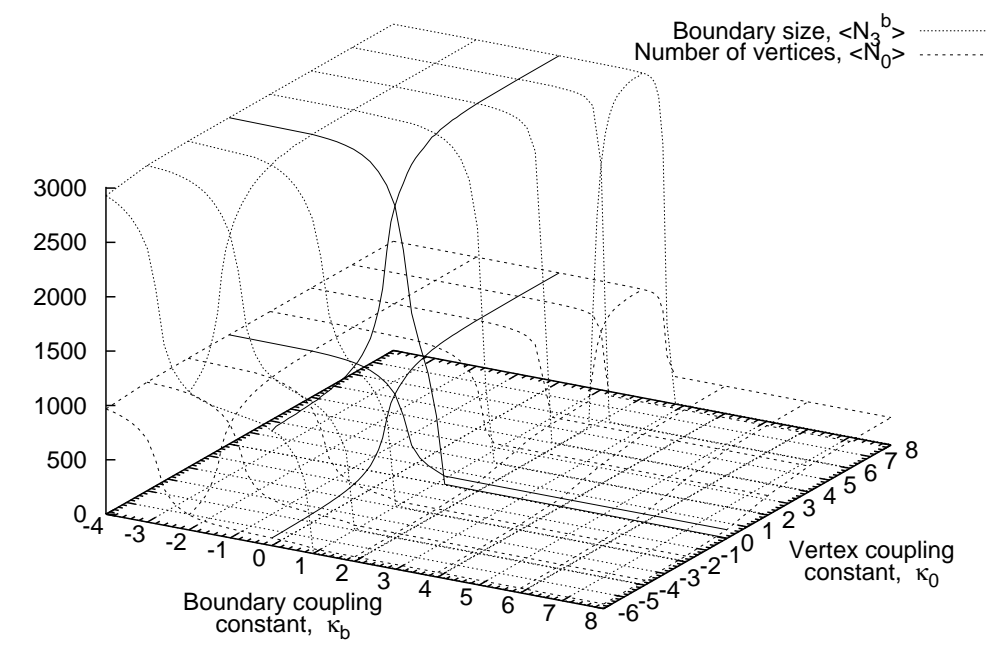

Figure 1: Boundary size $\left(\left\langle N_{3}^{b}\right\rangle\right)$ and number of vertices $\left(\left\langle N_{0}\right\rangle\right)$ for 4-dimensional dynamical triangulation with a boundary. Nominal simulation volume, $N_{4}=1000$.

'singular' vertex with $\left\langle q_{\max }\right\rangle=2642(6)$ (the next most coordinated vertex has $\left\langle q_{\text {nextMax }}\right\rangle=531(5)$, and $\left.\left\langle q_{\text {avg }}\right\rangle=250.7(5)\right)$

The presence of singular vertices is similar to the behaviour seen in compact triangulations in 4dimensions [0], 6]. In the compact case, there are two singular vertices which are equally coordinated. In simulations with a boundary we find one singular vertex and the minimal-boundary appears to assume the role of the other singular vertex (the number of simplices shared by boundary vertices is $1871(82))$.

We find $\left\langle d_{b d y}\right\rangle=9.18(4)$ (some correlation, $\tau=1.7$ ) and $\left\langle d_{a v g}\right\rangle=10.66(1)$. The approximate equality of these two distances tells us that in this phase the presence of the boundary plays no crucial role (except for changing the number of singular vertices). Furthermore, we have observed that the typical manifold is very crumpled, having only a logarithmic growth of its mean size. Such a behavior may be characterized by a large effective dimension and is reminiscent of the usual 


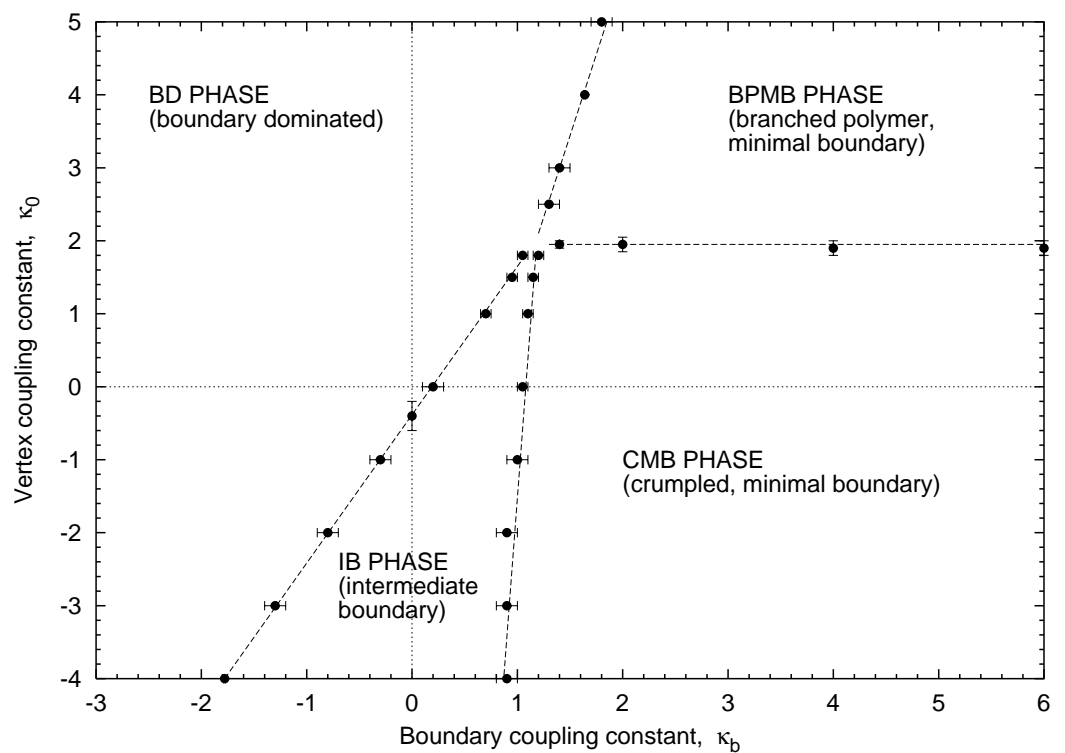

Figure 2: Phase diagram for 4-dimensional dynamical triangulation with a boundary. Nominal simulation volume, $N_{4}=1000$. Error bars are from estimation of the positions of the susceptibility peaks. Where error bars cannot be seen they are smaller than the symbols; the lines are guides to the eye.

crumpled phase seen in simulations of the sphere.

\section{BPMB phase}

Again we characterize the phase using data for $N_{4}=8000$ (116 samples have been acquired) with couplings $\kappa_{0}=5, \kappa_{b}=6$. We find $\left\langle d_{b d y}\right\rangle=43.3(10)$ and $\left\langle d_{a v g}\right\rangle=45.6(5)$. Again, the distance to the boundary is comparable to the distance to any randomly selected simplex indicating that the former has no distinguished role in the triangulation. Furthermore, large mean-geodesics are consistent with paths constrained to follow long branches. In this phase the vertex coordinations show no sign of singular structure: $\left\langle q_{\max }\right\rangle=387(7)$ and the next most highly coordinated vertex is about 50 lower, $\left\langle q_{a v g}\right\rangle=20.90(1)$. Thus, the physics of this phase is again rather like the corresponding situation on the sphere, the typical geometries are one-dimensional polymers. 


\section{BD phase}

In this phase the boundary is essentially maximal, the maximal value being obtained from an arbitrarily branched chain of simplices such that $N_{3 \text { maximal }}^{b}=3 \times N_{4}+2$. This is easy to understand in terms of a single chain of simplices - the 2 end 4 -simplices have 4 boundary 3 -simplices, and the $\left(N_{4}-2\right)$ middle 4 -simplices each have 3 boundary 3 -simplices. If one then considers adding a branch, one boundary simplex is lost at the branch point but one boundary simplex is gained at the end simplex, so there is no net change in $N_{3}^{b}$.

To support this we list data from a run at $N_{4}=8000$ and couplings $\kappa_{0}=-4, \kappa_{b}=-10$, in which we have collected 167 samples. We find that the boundary size fluctuates only very slightly about a mean of $N_{3}^{b} \approx 24002=3 * 8000+2$ and is very branched, possessing $\left\langle N_{\text {end }}\right\rangle=2534(2)$ end points. The maximum vertex coordination $\left\langle q_{\max }\right\rangle=103(2)$ is close to that expected for a flat lattice. Another indicator that the geometry corresponds to narrow tubes is seen when we examine the geodesic distances: $\left\langle d_{b d y}\right\rangle=0.5038(2)$ and $\left\langle d_{a v g}\right\rangle=125.8(2)$ The mean boundary distance tells us that every simplex is a boundary simplex. Thus the quantum geometry is effectively onedimensional. We find $\left\langle q_{a v g}\right\rangle=4.9975000$ (1) which we take as further indication that there are no sections of bulk in this phase. The argument for this is as follows: in a minimal-width chain with no branches we have two ends which have a few vertices shared by less than 5 -simplices - the coordination of vertices in the bulk of the chain. At each end there is 1 vertex with coordination 1, 1 with coordination 2, 1 with coordination 3 , and 1 with coordination 4 . This adds up to a coordination deficit of 10 for the 4 vertices at an end, 20 for both ends. In a minimal-width chain consisting of $N_{4}$ simplices we expect $N_{0}=N_{4}+4$ and so with $N_{4}=8000$ we expect, and see, $N_{0}=8004$. This allows us to calculate the expected $\left\langle q_{a v g}\right\rangle=\left(N_{0} \times 5-20\right) / N_{0}$, putting $N_{0}=8004$ we get $\left\langle q_{a v g}\right\rangle=4.997501$. Adding branches to the chain results in more highly coordinated vertices at the branch point that exactly cancel the deficit from the additional end. 


\section{IB phase}

Here we show data for $N_{4}=8000$ (86 samples) at couplings $\kappa_{0}=-4, \kappa_{b}=0$. We find that, once again, the boundary size scales linearly with the volume (see figure 3) yielding $N_{3}^{b} \approx N_{4} \times 1.033$. We also find one large singular vertex, with $\left\langle q_{\max }\right\rangle=5721(9)\left(\left\langle q_{\text {nextMax }}\right\rangle=233(2),\left\langle q_{\text {avg }}\right\rangle=133(1)\right)$. The measurements of geodesic distances show that the boundary plays a preferred role in the triangulation $\left\langle d_{b d y}\right\rangle=0.834(2)$ while $\left\langle d_{a v g}\right\rangle=11.36(1)$. These numbers are incompatible with an extremal branched polymer shape. However, these measurements by themselves would not be inconsistent with a 'fat-branch' model. If we add to this the presence of a single singular vertex and the observation that about 40\% (3495 (9) out of 8000) of the simplices have one boundary face we tentatively conclude that the typical geometry in this phase is three-dimensional - the boundary of the system coinciding with the boundary of the 4-ball surrounding the singular vertex.

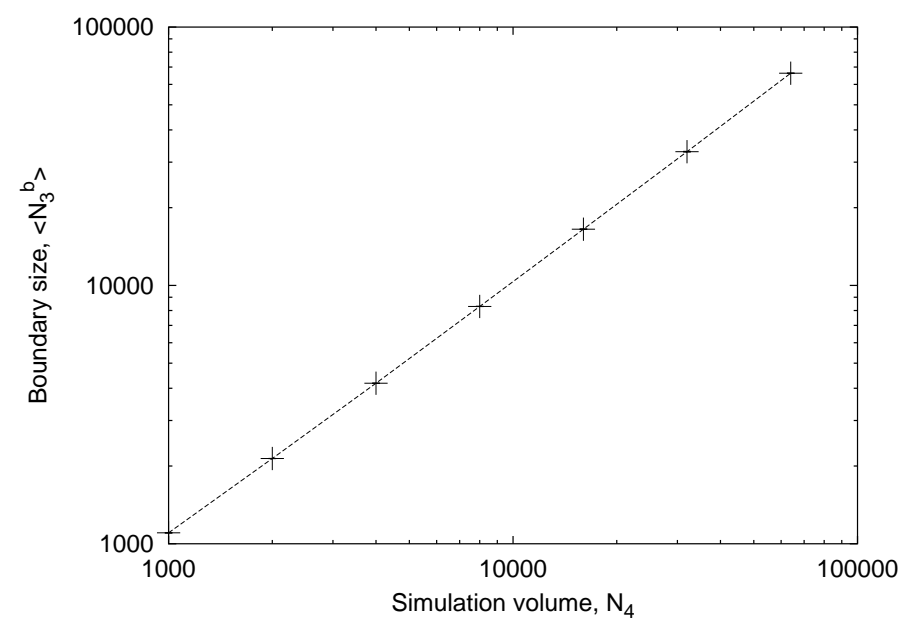

Figure 3: Plot showing scaling of boundary size with simulation volume for the intermediate boundary (IB) phase, $\kappa_{0}=-4, \kappa_{b}=0$. The error bars are much smaller than the symbols. 


\section{Phase transitions}

The phase transition between the CMB and BPBM phases has been studied in the simulations of compact systems in three and four-dimensions and found to be discontinuous (first-order) in both cases (3d [3], 4d औ, 8]). Our simulations have minimal boundary in both phases and so we expect the same behaviour. This was verified in three dimensions [11].

We have also investigated the transition between CMB and IB phases and find good evidence here also for a discontinuous phase transition (figure 4 , top; $N_{4}=1000, \kappa_{0}=-4, \kappa_{b}=0.9$ ). Similarly, the time series close to the BPMB-BD phase boundary (figure 4 , middle; $N_{4}=1000, \kappa_{0}=4$, $\left.\kappa_{b}=1.64\right)$ also shows signs of bistability indicative of a discontinuous transition.

At $N_{4}=1000,2000$ and 4000 we found no such signals near the BD - IB boundary. However, we found linear scaling of the height of the peak in the boundary-size susceptibility which indicates a first-order transition. To confirm this we extended our simulations to $N_{4}=8000$ and found bistability in the time series (figure 1 , bottom; $N_{4}=8000, \kappa_{0}=-4, \kappa_{b}=-1.862$ ).

\section{Concluding remarks}

We have simulated four-dimensional simplicial gravity on manifolds with the topology of a 4disk. Our action contains both bulk curvature terms and a boundary cosmological constant term. We have identified four phases in the model within the range of couplings $-6<\kappa_{0}<6$ and $-4<\kappa_{b}<8$. The observed phases include the crumpled and branched-polymer phases seen in triangulations of compact manifolds, and the boundary dominated phase seen in three-dimensions. The latter consists of a maximally branching tree. We have also identified a fourth phase which resembles a singular 4-ball in which essentially all simplices share a common bulk vertex and the physics is dominated by the three-dimensional boundary.

All of the boundaries between these phases appear to be associated with discontinuous phase 

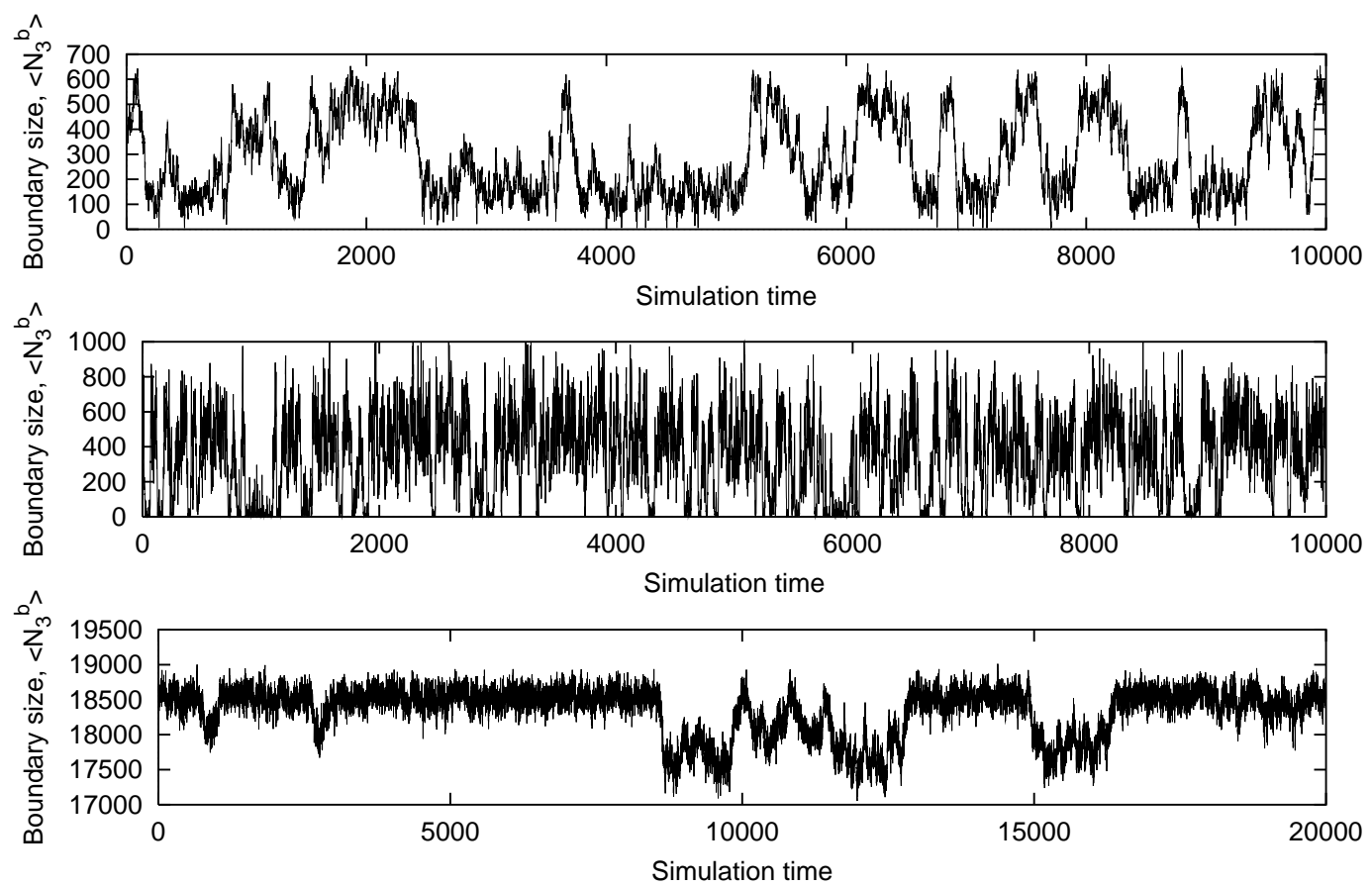

Figure 4: Simulation time series showing bistability in the boundary size $\left(N_{3}^{b}\right)$ at the CMB - IB phase boundary (upper; $N_{4}=1000, \kappa_{0}=-4, \kappa_{b}=0.9$ ); the BPMB - BD phase boundary (middle; $N_{4}=1000, \kappa_{0}=4, \kappa_{b}=1.64$ ); and the BD-IB phase boundary (lower; $N_{4}=8000, \kappa_{0}=-4$, $\left.\kappa_{b}=-1.862\right)$. We take this as indication of the discontinuous nature of these transitions.

transitions. This is the same situation as for four-dimensional simplicial gravity on manifolds with $S^{4}$ topology and means that we cannot take a continuum limit in the vicinity of any of the observed phase transitions. Notice that all four phase boundaries appear to meet (within our errors) at one unique point. The simplest explanation for this feature is to assume that our phase diagram contains only two independent transition lines which would then generically intersect at a single point. Glancing at the nature of the phases one would associate one line with a boundary dividing lattices with singular vertices from those without. The second line would then correspond to a dividing line between geometries with extended boundary and those with minimal boundary.

Notice also, that in all the phases we have identified, the number of vertices on the boundary is strongly correlated with the boundary volume. This need not be the case and it would be interesting 
to vary the coupling $\kappa_{b}^{0}$ away from zero to see whether a phase could be found which exhibited a classical scaling of boundary size with 4-volume.

\section{Acknowledgments}

Simon Catterall was supported in part by DOE grant DE-FG02-85ER40237. Simeon Warner was supported in part by the DOE under contract W-7405-ENG-36.

\section{References}

[1] E Adi, M Hasenbusch, M Marcu, E Pazy, K Pinn and S Solomon. Monte carlo simulation of 2-d quantum gravity as open dynamically triangulated random surfaces. Physiscs Letters B, $320227-233$ (1994). arXiv:hep-lat/9310016.

[2] J Ambjorn. Quantization of geometry. (1994). arXiv:hep-th/9411179.

[3] J Ambjorn, D V Boulatov, A Krzywicki and S Varsted. The vacuum in three-dimensaional simplicial quantum gravity. Physiscs Letters B, 276 432-436 (1992).

[4] P Bialas, Z Burda, A Krzywicki and B Petersson. Focusing on the fixed point of 4d simplicial gravity. Nuclear Physics B, 472 293-308 (1996). arXiv:hep-lat/9601024.

[5] S Catterall. Simulations of dynamically triangulated gravity - an algoritm for arbitrary dimension. Computer Physics Communications, 87 409-415 (1995). arXiv:hep-lat/9405026.

[6] S Catterall, R Renken and J Kogut. Singular structure in 4d simplicial gravity. PhysLettB, 416 274-280 (1998). arXiv:hep-lat/9709007.

[7] S Catterall, G Thorleifsson, J Kogut and R Renken. Singular vertices and the triangulation space of the d-sphere. Nuclear Physics B, 468 263-276 (1996). arXiv:hep-lat/9512012. 
[8] B de Bakker. Further evidence that the transition of $4 \mathrm{~d}$ dynamical triangulation is $1^{s} t$ order. Physiscs Letters B, 389 238-242 (1996). arXiv:hep-lat/9603024.

[9] J B Hartle and R Sorkin. Boundary terms in the action for the regge calculus. General Relativity and Gravity, 13 541-549 (1981).

[10] Nicholas Metropolis, Arianna W Rosenbluth, Marshall N Rosenbluth, Augusta H Teller and Edward Teller. Equation of state calculations by fast computing machines. The Journal of Chemical Physics, 21 (1953).

[11] Simeon Warner, Simon Catterall and Ray Renken. Phase diagram of three-dimensional dynamical triangulations with a boundary. Physiscs Letters B, 442 266-272 (1998). arXiv:hep-lat/9808006. 